\title{
Surveillance de la santé mentale positive et de ses facteurs déterminants au Canada : élaboration d'un cadre d'indicateurs de surveillance de la santé mentale positive
}

\author{
H. Orpana, Ph. D. (1,2); J. Vachon, M. Sc. (1); J. Dykxhoorn, M. Sc. (1,3); L. McRae, B. Sc. (1); G. Jayaraman, Ph. D. (1,3)
}

Cet article a fait l'objet d'une évaluation par les pairs.

Diffuser cet article sur Twitter

\section{Résumé}

Introduction : D’après la Stratégie en matière de santé mentale pour le Canada, la collecte de données sur la santé mentale au Canada a besoin d'être améliorée. La mise en place de systèmes de surveillance sur la maladie mentale n’a pas suffi à combler toutes les lacunes statistiques relevant de la santé mentale positive et de ses facteurs déterminants au Canada. L’objectif de ce projet était d'élaborer un Cadre d'indicateurs de surveillance de la santé mentale positive, dans le but de dresser un portrait de la santé mentale positive au Canada, ainsi que de ses facteurs déterminants. Les données recueillies grâce à ce cadre de surveillance serviront de référence pour l'établissement des programmes et des politiques d'amélioration de la santé mentale des Canadiens.

Méthodologie : Un examen de la littérature et une analyse contextuelle ont été réalisés afin de fournir une base théorique au cadre et de sélectionner divers critères d'évaluation de la santé mentale positive et de ses facteurs de risque et de protection. La définition de la santé mentale positive adoptée par l'Agence de la santé publique du Canada a servi de référence conceptuelle lors de l'élaboration du cadre. À partir de la liste exhaustive des risques et des facteurs de protection qui a été réalisée, des experts en santé mentale, des partenaires gouvernementaux et des intervenants indépendants ont été invités à classer les indicateurs choisis par ordre de priorité. Ces groupes ont ensuite été invités à sélectionner les méthodes de mesure les plus prometteuses pour chacun des indicateurs.

Résultats : Le cadre conceptuel de surveillance de la santé mentale positive et de ses facteurs déterminants qui a été ainsi élaboré contient 5 indicateurs de résultats et 25 indicateurs de déterminants, répartis en 4 niveaux contextuels: «individu», «famille », « communauté» et «société». Ce cadre d'indicateurs vise à combler les lacunes en matière de données relevées par la Stratégie en matière de santé mentale pour le Canada, et il servira de référence pour l'établissement des programmes et des politiques d'amélioration de la santé mentale des Canadiens tout au long de leur vie.

Mots-clés : indicateurs de l'état de santé, santé mentale

\section{Introduction}

L’Agence de la santé publique du Canada (l’Agence) définit la santé mentale positive comme «la capacité qu'a chacun d'entre nous de ressentir, de penser et d'agir de manière à améliorer notre aptitude à jouir de la vie et à relever les défis auxquels nous sommes confrontés. Il s'agit d'un sentiment positif de bien-être émotionnel et spirituel qui respecte l'importance de la culture, de l'équité, de la justice sociale, des interactions et de la dignité personnelle ${ }^{1}$. De même, l'Organisation mondiale de la santé (OMS)
Principales constatations

- L’Agence de la santé publique du Canada a élaboré un cadre conceptuel de surveillance de la santé mentale positive et de ses facteurs déterminants au Canada.

- 5 indicateurs de résultats et 25 facteurs déterminants ont été sélectionnés et répartis en 4 niveaux contextuels : individu, famille, communauté et société.

- Le cadre fournit une structure à la collecte des données de surveillance de la santé mentale positive qui serviront de référence pour l'élaboration de programmes et politiques en matière de promotion de la santé des Canadiens tout au long de leur vie.

- Le cadre comble ainsi les principales lacunes dans les données relevées dans la Stratégie en matière de santé mentale pour le Canada, Changer les orientations, changer des vies.

définit la santé mentale comme « un état de bien-être qui permet à chacun de réaliser son potentiel, de faire face aux difficultés normales de la vie, de travailler avec succès et de manière productive et d'être en mesure d'apporter une contribution à la communauté $»^{2}$. La dimension positive de la santé mentale est mise en lumière par la définition de la santé dans la constitution de l'OMS : «La santé est un état de complet bien-être physique, mental et social et ne consiste pas seulement en une absence de maladie ou d'infirmité » ${ }^{2}$.

Rattachement des auteurs :

1. Division de la surveillance et de l'épidémiologie, Centre de prévention des maladies chroniques, Agence de la santé publique du Canada, Ottawa (Ontario), Canada

2. École de psychologie, Université d'Ottawa, Ottawa (Ontario), Canada

3. Département d'épidémiologie et de médecine communautaire, Université d'Ottawa, Ottawa (Ontario), Canada

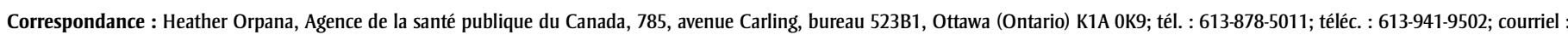
heather.orpana@phac-aspc.gc.ca 
La surveillance de la santé publique, l'une des six fonctions fondamentales liées à la santé publique $^{3}$, est définie comme [traduction] « la collecte continue et systématique, l'analyse et l'interprétation de données de santé essentielles pour la planification, la mise en place et l'évaluation des pratiques en santé publique $»^{4}$. Les programmes de surveillance de l'Agence font le suivi d'un large éventail de sujets liés aux maladies chroniques, aux blessures et aux comportements en matière de santé, dont la santé mentale et le suicide, au sein de la population canadienne. Dans la Stratégie en matière de santé mentale pour le Canada, Changer les orientations, changer des vies ${ }^{5}$, on recommande « [d'a]méliorer la collecte de données et les recherches qui permettront de mieux comprendre les besoins en matière de santé mentale et les forces des divers groupes au sein de la population » ${ }^{5, p .85}$ et «[d'a]méliorer la collecte de données, la recherche et l'échange de connaissances [en matière de santé mentale] partout au Canada »" p.124. Dans le cadre du budget fédéral de 2013, le gouvernement du Canada a ordonné la réattribution de 2 millions de dollars supplémentaires par année sur trois ans pour améliorer la collecte de données et les connaissances et de favoriser la collaboration en matière de santé mentale. Quoique l'Agence bénéficie d'un système de surveillance de la maladie mentale bien établi ${ }^{6}$, il n'existait à ce jour aucun système de surveillance axé sur la santé mentale positive des Canadiens. Pour combler cette lacune, l'Agence a entrepris, avec la collaboration d'experts et d'intervenants clés, d'élaborer un cadre conceptuel et une série d'indicateurs de base pour la surveillance de la santé mentale positive et de ses facteurs déterminants. Les indicateurs seront utiles pour l'élaboration de programmes et de politiques visant à améliorer l'état de santé mentale des Canadiens tout au long de leur vie. Bien que le cadre soit destiné en priorité aux professionnels de la santé publique, aux promoteurs de politiques et de programmes et aux décideurs, nous nous attendons à ce que le public s'y intéresse, étant donnée l'attention grandissante portée à la santé mentale positive et au bien-être.

Dans cet article, nous décrivons le processus entrepris par l'Agence pour établir un cadre d'indicateurs de surveillance de la santé mentale positive, nous présentons la logique et les principes qui sous-tendent le projet et nous détaillons son état d'avancement. Nous traitons à la fois du cadre conceptuel et des indicateurs de base choisis pour la surveillance.

\section{Cadre conceptuel}

Afin de faire le bilan des cadres de surveillance de la santé mentale déjà en place, un bibliothécaire a effectué une recherche documentaire dans Embase (de 1974 à 2013), Medline (de 1946 à 2013) et PsycINFO à partir des mots-clés suivants, seuls ou en association : mental health, mental disorders, indicators, criteria, method, measure, policy, policies, develop, surveillance, taxonomy, framework, performance, health status indicators, quality indicators et health care. Les résultats ont été limités aux articles en français et en anglais, et ont été exclus les articles cliniques sur une population de patients en particulier. Au total, 88 articles ont été sélectionnés. Une recherche complémentaire en ligne a été faite avec Google et les mots-clés mental health, surveillance et framework.

Bien qu'aucun cadre de surveillance ne portât exclusivement sur la santé mentale positive et que la plupart aient surtout comme objet la maladie mentale, diverses composantes de cadre ont été dégagées, notamment dans Waddell et collab. ${ }^{\text {, }}$ Parkinson $^{8}$ et Korkeila et collab. ${ }^{9}$. En outre, nous avons analysé les démarches en matière de santé publique et de promotion de la santé susceptibles de fournir une structure organisationnelle socioécologique au cadre $\mathrm{c}^{10-12}$.

Cette recherche a permis de créer un cadre conceptuel fournissant un fondement théorique au projet, grâce à la collaboration d'experts de la Commission de la santé mentale du Canada (CSMC). Les administrateurs et le Conseil consultatif de la CSMC l'ont ensuite revu. Ce cadre regroupe les éléments nécessaires à la description de la santé mentale positive au sein de la population (figure 1).

La figure 2 résume les étapes de l'élaboration du Cadre d'indicateurs de surveillance de la santé mentale positive. Quatre composantes ont été réunies dans un cadre conceptuel général, qui a ensuite servi de base pour la sélection des indicateurs.
Premièrement, la santé mentale positive a été définie comme un état de bien-être que toute personne, qu'elle soit atteinte de maladie mentale ou non, est en mesure d'améliorer ${ }^{5}$. Le concept de santé mentale positive s'appliquant à tout le monde, on peut envisager que son mécanisme assure une meilleure répartition du bien-être, de ce fait accessible à l'ensemble de la population.

Deuxièmement, divers facteurs de risque et de protection, ou déterminants de la santé mentale positive, ont été sélectionnés pour nourrir ce cadre, et ce sont eux qui forment la cible des efforts d'intervention et d'amélioration en regard de la santé mentale positive de la population ${ }^{13}$.

Troisièmement, un modèle socioécologique présentant les niveaux contextuels où se retrouvent ces facteurs de risque et de protection a été ajouté au cadre conceptuel ${ }^{8-11}$. Ces niveaux contextuels, qui sont ceux de l'individu, de la famille, de la communauté et de la société, sont présentés dans la figure 1. Chacun a une incidence sur la santé mentale positive de la population et constitue un point d'entrée possible pour les interventions de promotion de la santé mentale.

Quatrièmement, on a tenu compte du parcours de vie dans l'élaboration du cadre conceptuel, étant donné que les facteurs de risque et de protection varient et s'accumulent et que les expériences vécues dans l'enfance peuvent influencer la santé mentale positive plus tard au cours de l'existence ${ }^{14}$. Le parcours de vie a ainsi été divisé en trois périodes : l'enfance (0 à 11 ans), la jeunesse (12 à 17 ans) et l'âge adulte (18 ans et plus). Bien que ces grandes catégories soient hétérogènes, il a été décidé de maintenir des seuils assez généraux dans le parcours de vie, quitte à apporter des précisions grâce à des indicateurs et mesures spécifiques. En fait, tous les indicateurs sauf quatre sont les mêmes pour les différentes périodes de vie, alors que les mesures choisies pour les indicateurs diffèrent selon la période de vie.

\section{Critères de sélection des indicateurs}

Une fois le cadre conceptuel établi, des indicateurs et des mesures ont été attribués à chaque niveau. Les indicateurs ont été 
FIGURE 1

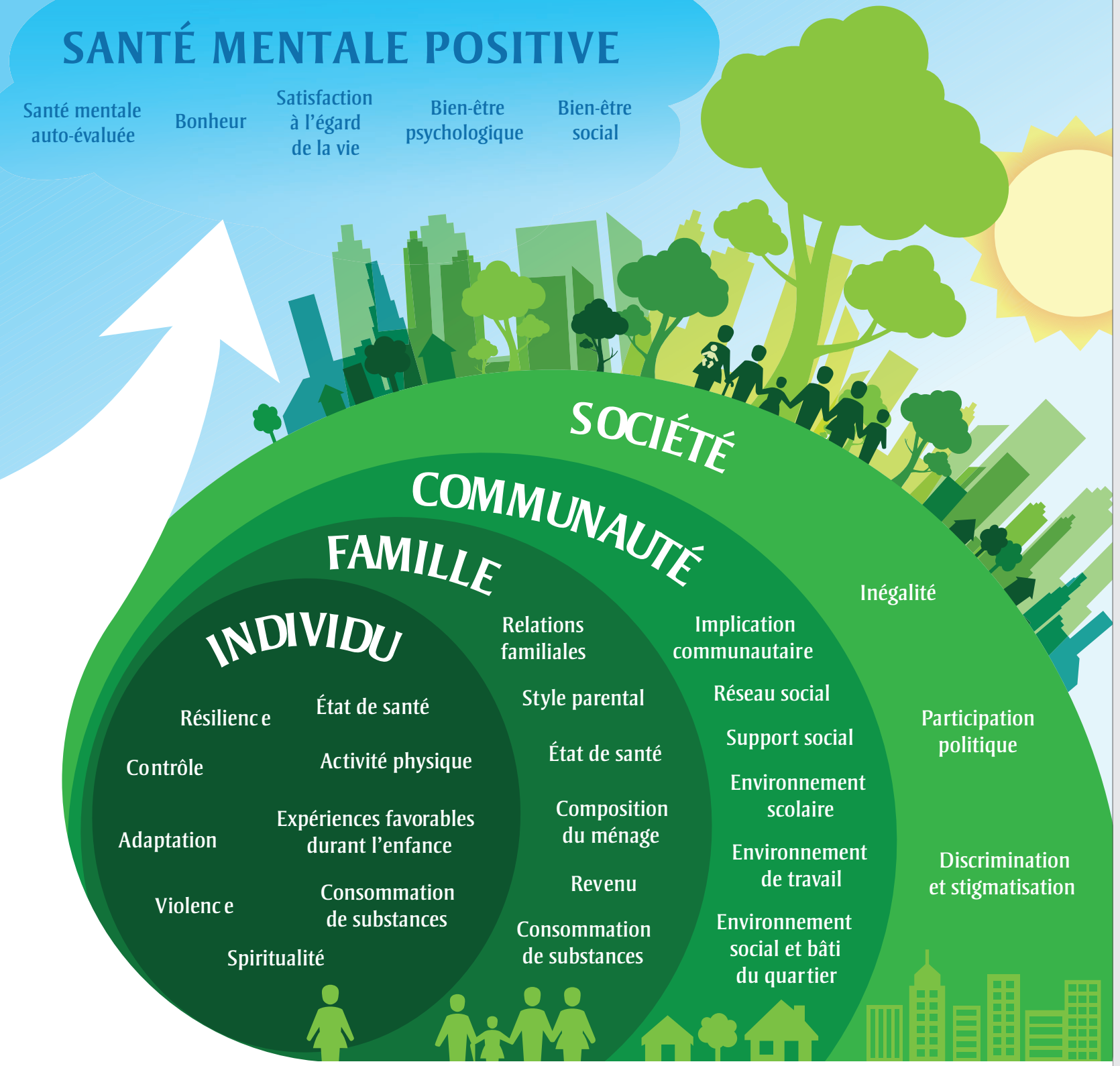

La santé mentale positive est importante pour tous, et ce, même pour les Canadiens qui vivent avec une maladie mentale.

définis comme des éléments pouvant être mesurés et faisant l'objet de comptes rendus. Les mesures, quant à elles, permettent de rendre opérationnels les indicateurs à l'aide de questions de sondage, d'échelles ou d'autres méthodes.
Cinq critères de sélection (pertinence, exploitabilité, exactitude, faisabilité et stabilité) ont servi à hiérarchiser les indicateurs et les mesures de santé mentale positive (tableau 1). Les définitions adoptées pour ces critères sont largement répandues pour évaluer des indicateurs ${ }^{15}$. Nous avons également choisi ces critères par souci d'uniformité avec les critères de sélection du Cadre conceptuel d'indicateurs des maladies chroniques ${ }^{16}$ ainsi que de cadres d'indicateurs internationaux ${ }^{17-20}$. 
FIGURE 2

Processus d'élaboration d'un cadre d'indicateurs de surveillance de la santé mentale positive

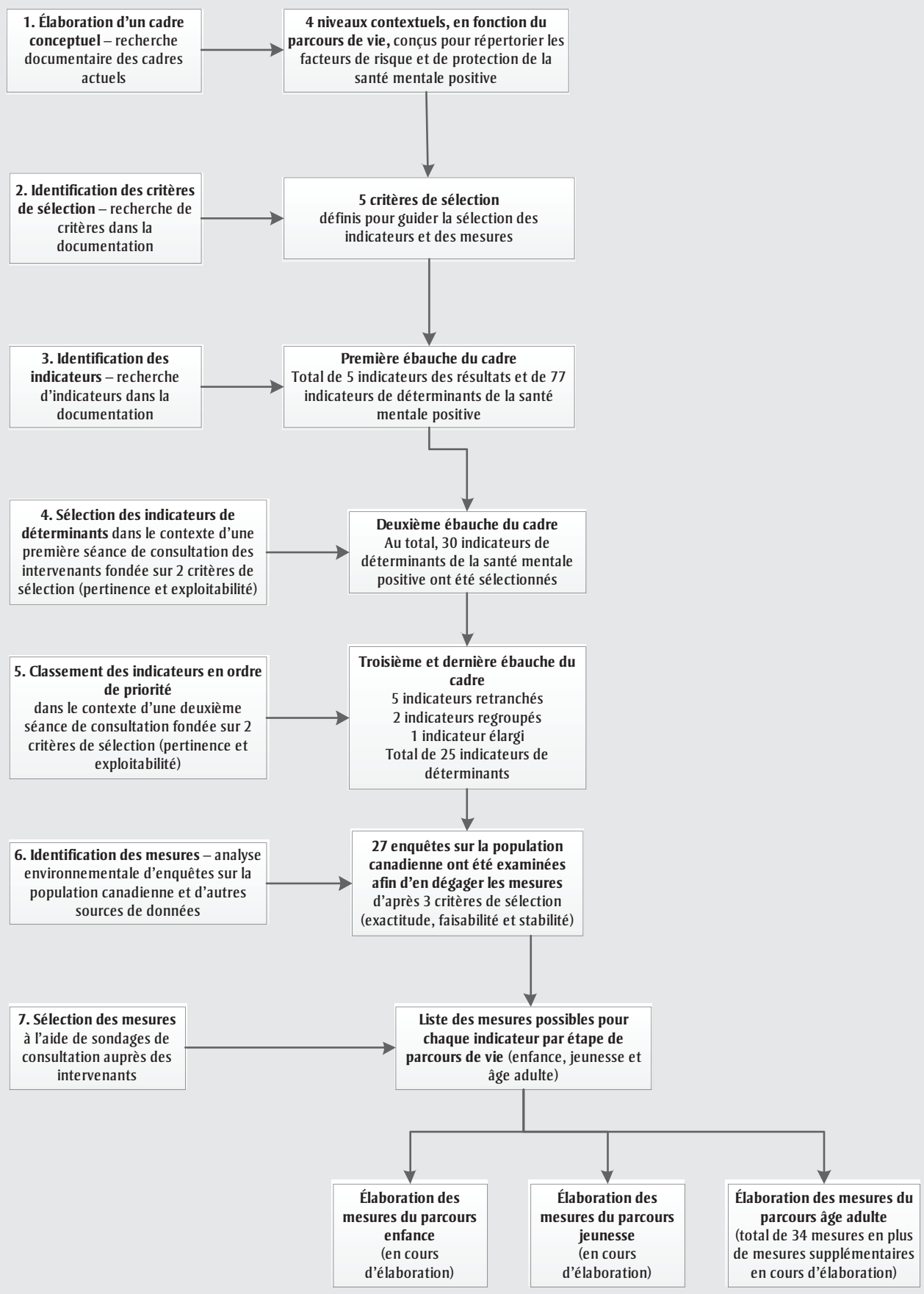

La pertinence et l'exploitabilité ont été examinées dans le contexte de la politique et des programmes de santé publique. L'exactitude, la faisabilité et la stabilité des données ont été évaluées dans le contexte des programmes de surveillance susceptibles de recueillir ces données. Ces critères ont servi à la fois à sélectionner les indicateurs, à les classer en ordre de priorité et à leur assortir des mesures.

\section{Sélection des indicateurs}

Nous avons dressé, à partir de la documentation consultée, une liste exhaustive des indicateurs potentiels pour un cadre de surveillance de la santé mentale positive (Figure 1). Nous avons consulté au besoin d'autres documents pertinents portant sur le contenu de certaines composantes spécifiques, notamment les résultats en matière de santé mentale positive.

Nous avons commencé par sélectionner les indicateurs fournissant des résultats fondés 
TABLEAU 1

Critères de sélection des indicateurs et des mesures

\begin{tabular}{|c|c|}
\hline $\begin{array}{l}\text { Critères de } \\
\text { sélection }\end{array}$ & Description \\
\hline Pertinence & Fournit des renseignements significatifs et pertinents à l'utilisateur cible ${ }^{16-18}$. \\
\hline Exploitabilité & $\begin{array}{l}\text { Fournit des renseignements susceptibles d'informer, d'influencer ou de changer les } \\
\text { pratiques et les politiques en matière de santé publique }{ }^{16-18} \text {. }\end{array}$ \\
\hline Exactitude & $\begin{array}{l}\text { Correspond aux meilleures données probantes. Robuste sur le plan scientifique, valable, } \\
\text { fiable, sensible aux variations, interprétable et complet }{ }^{16-18} \text {. }\end{array}$ \\
\hline Faisabilité & $\begin{array}{l}\text { Données disponibles et de qualité suffisante, ou collecte de données pouvant être mise } \\
\text { en place à un coût relativement faible }{ }^{16-18} \text {. }\end{array}$ \\
\hline Stabilité & Les données sont recueillies régulièrement et sont comparables dans le temps ${ }^{16-18}$. \\
\hline
\end{tabular}

sur la théorie contemporaine du bien-être et de la santé mentale positive, qui, en général, dégage deux composantes : l'hédonie, ou bien-être émotionnel, et l'eudémonie, ou fonctionnement positif ${ }^{21}$. L'hédonie est représentée par des mesures de sentiments positifs et de satisfaction à l'égard de la vie (bien-être émotionnel), alors que l'eudémonie implique un bon fonctionnement, par exemple la capacité de participer à des activités gratifiantes et d'entretenir des relations significatives (bien-être psychologique et social) ${ }^{22}$. Ces résultats devaient également s'harmoniser avec la définition opérationnelle de la santé mentale positive adoptée par l'Agence ${ }^{23}$.

Nous avons ensuite sélectionné des indicateurs des déterminants de la santé mentale positive, capables de rendre compte des facteurs de risque et de protection de la santé mentale positive dans les quatre niveaux contextuels définis : individu, famille, communauté et société. Nous avons choisi un certain nombre d'indicateurs dans la documentation et dans d'autres cadres d'indicateurs en santé mentale (en particulier Waddell et collab. ${ }^{7}$, Parkinson ${ }^{8}$ et Korkeila et collab. ${ }^{9}$ ). Une synthèse thématique des indicateurs a permis le regroupement des éléments similaires afin de simplifier le cadre et de le rendre plus compréhensible. Chaque indicateur a fait l'objet d'une définition claire et concise et d'une justification basée sur des données probantes faisant état d'un lien entre chaque déterminant et la santé mentale positive.

Une première liste de 5 indicateurs de résultats et de 77 indicateurs de déterminants potentiels en matière de santé mentale positive a été dressée (tableau 2). Ensuite, en nous appuyant sur l'expérience antérieure de l'Agence de l'utilisation d'un processus Delphi modifié pour la sélection des indicateurs du Cadre conceptuel d'indicateurs des maladies chroniques ${ }^{16}$ et pour l'élaboration d'indicateurs nationaux pour la surveillance de l'ostéoporose au Canada ${ }^{24}$, consultation itérative pour suivre une démarche structurée aboutissant à la sélection d'indicateurs tout en tenant compte des points de vue et des besoins des différents groupes d'intervenants. L'objectif premier des données de surveillance étant d'éclairer les mesures de santé publique, on a considéré que ces intervenants étaient les professionnels de la santé publique travaillant dans le domaine de la surveillance, des programmes ou des politiques en matière de santé mentale, tant à l'interne qu'à l'externe.

La première liste de 77 indicateurs de déterminants a ainsi été réduite, grâce à deux processus de consultation itératifs.

Tout d'abord, le Comité aviseur sur la surveillance de la santé mentale et des maladies mentales, un groupe d'experts canadiens conseillant l'Agence sur l'élaboration, l'utilisation et l'évaluation de renseignements de surveillance de la santé mentale et des maladies mentales, a été convié à une rencontre en personne en janvier 2014. Le comité est composé de membres du milieu universitaire, d'organismes nationaux et de gouvernements provinciaux et territoriaux. Les 10 membres du comité ont été répartis en deux groupes pour des séances en plus petit comité : 5 membres ont traité des indicateurs du niveau «individu», les 5 autres des niveaux «famille », « communauté » et « société ». Chaque groupe a examiné la nous avons mis au point un processus de première liste d'indicateurs, discuté des concepts sous-tendant chaque indicateur et fourni des commentaires sur la réorganisation des indicateurs et l'établissement des priorités. Chaque groupe a ensuite rendu compte de ses constatations et de ses décisions à l'ensemble du comité. En se fondant sur deux des critères de sélection (pertinence et exploitabilité), le comité est parvenu à un consensus sur les principaux résultats en matière de santé mentale positive et sur les cinq premiers indicateurs de déterminants dans chaque niveau. La liste des indicateurs de déterminants a été réduite aux 30 indicateurs les plus pertinents et les mieux exploitables associés à une santé mentale positive.

Ensuite, le Groupe de travail sur la promotion de la santé mentale, qui relève du Comité directeur pour des personnes et des communautés en santé, a participé à la seconde phase de consultation. Ce comité directeur est l'un des trois comités directeurs (fédéral, provincial et territorial) du Réseau pancanadien de santé publique ${ }^{25}$. Le groupe de travail était composé d'experts en promotion de la santé mentale relevant de plusieurs instances provinciales et territoriales, de même que de représentants de l'Agence et de la Direction générale de la santé des Premières nations et des Inuits de Santé Canada.

Comme lors de la première phase de consultation, les concepts sous-tendant chaque indicateur et les données probantes sur les liens entre facteurs de risque et de protection et résultats en matière de santé mentale positive ont fait l'objet de discussions. On a invité les 11 représentants du groupe de travail à vérifier si certains des 30 indicateurs de déterminants de la liste révisée étaient redondants et si certains autres indicateurs manquaient, puis à classer l'ensemble des indicateurs choisis en ordre de priorité. Pour cela, on leur a demandé d'utiliser une technologie de vote par internet, afin qu'ils sélectionnent les 5 indicateurs les plus pertinents et les mieux exploitables selon eux pour le niveau « individu » (sur 12 possibles), 3 pour le niveau «famille» (sur 7 possibles) et 4 pour le niveau «communauté » (sur 8 possibles). Le niveau «société » ne faisait pas partie de la sélection car il ne comptait que 3 indicateurs. Chaque indicateur choisi 
TABLEAU 2

Liste initiale et liste finale des indicateurs de surveillance de la santé mentale positive

\begin{tabular}{|c|c|}
\hline Liste initiale d'indicateurs possibles & Liste finale des indicateurs \\
\hline \multicolumn{2}{|l|}{ A. RÉSULTATS EN SANTÉ MENTALE POSITIVE } \\
\hline $\begin{array}{l}\text { 1.1. Bien-être hédonique } \\
\text { a. Bien-être subjectif } \\
\text { b. Bonheur (émotions positives) } \\
\text { c. Satisfaction à l'égard de la vie } \\
\text { d. Bien-être émotionnel }\end{array}$ & $\begin{array}{l}\text { 1. Auto-évaluation de la santé mentale } \\
\text { 2. Bonheur } \\
\text { 3. Satisfaction à l'égard de la vie }\end{array}$ \\
\hline $\begin{array}{l}\text { 1.2. Bien-être eudémonique } \\
\text { a. Bien-être psychologique }\end{array}$ & $\begin{array}{l}\text { 4. Bien-être psychologique } \\
\text { 5. Bien-être social }\end{array}$ \\
\hline \multicolumn{2}{|l|}{$\begin{array}{l}\text { B. FACTEURS DE RISQUE ET DE PROTECTION } \\
\text { 1. INDIVIDU }\end{array}$} \\
\hline $\begin{array}{l}\text { 1.1. État de santé général } \\
\text { a. Auto-évaluation de la santé } \\
\text { b. Auto-évaluation de la santé mentale } \\
\text { c. Présence de problèmes de santé chroniques }\end{array}$ & $\begin{array}{l}\text { 6. État de santé } \\
\text { (Auto -évaluation de la santé mentale pour les mesures) }\end{array}$ \\
\hline $\begin{array}{l}\text { 1.2. Habitudes de santé personnelles } \\
\text { a. Hygiène de vie / habitudes de santé personnelles } \\
\text { b. Activité physique } \\
\text { c. Activité sédentaire } \\
\text { d. Habitudes alimentaires } \\
\text { e. Indice de masse corporelle }\end{array}$ & 7. Activité physique \\
\hline $\begin{array}{l}\text { 1.3. Toxicomanie et comportements à risque pour la santé } \\
\text { a. Consommation de tabac/tabagisme } \\
\text { b. Consommation d'alcool/alcoolisme } \\
\text { c. Consommation de drogues/toxicomanie } \\
\text { d. Mesures de prévention des blessures } \\
\text { e. Habitudes sexuelles à risque } \\
\text { f. Jeu compulsif }\end{array}$ & 8. Consommation problématique de substances (drogues/alcool) \\
\hline $\begin{array}{l}\text { 1.4. Croissance et développement } \\
\text { a. Alimentation de la mère } \\
\text { b. Prise de compléments alimentaires pendant la grossesse } \\
\text { c. Allaitement } \\
\text { d. Consommation d'alcool pendant la grossesse } \\
\text { e. Tabagisme pendant la grossesse et l'allaitement } \\
\text { f. Consommation de substances pendant la grossesse } \\
\text { g. Exposition à des risques pendant l'enfance }\end{array}$ & 3. Expériences favorables dans l'enfance \\
\hline $\begin{array}{l}\text { 1.5. Patrimoine biologique et génétique } \\
\text { a. Patrimoine biologique et génétique }\end{array}$ & \\
\hline $\begin{array}{l}\text { 1.6. Personnalité } \\
\text { a. Estime de soi } \\
\text { b. Sentiment de maîtrise } \\
\text { c. Sentiment de cohérence } \\
\text { d. Optimisme/pessimisme } \\
\text { e. Intelligence émotionnelle }\end{array}$ & $\begin{array}{l}\text { 1. Résilience } \\
\text { 4. Maîtrise de soi et auto -efficacité }\end{array}$ \\
\hline $\begin{array}{l}\text { 1.7. Spiritualité et religiosité } \\
\text { a. Spiritualité } \\
\text { b. Religiosité }\end{array}$ & 9. Spiritualité \\
\hline $\begin{array}{l}\text { 1.8. Expériences négatives pendant l'enfance } \\
\text { a. Expériences négatives pendant l'enfance }\end{array}$ & \\
\hline $\begin{array}{l}\text { 1.9. Stress de la vie courante } \\
\text { a. Violence (violence familiale, maltraitance, abus) } \\
\text { b. Discrimination } \\
\text { c. Contraintes financières/gestion des dettes }\end{array}$ & $\begin{array}{l}\text { 5. Violence } \\
\text { (Discrimination, dans « Société ») }\end{array}$ \\
\hline $\begin{array}{l}\text { 1.10. Adaptation } \\
\text { a. Adaptation }\end{array}$ & 2. Adaptation \\
\hline
\end{tabular}

Suite page suivante 
TABLEAU 2 (suite)

Liste initiale et liste finale des indicateurs de surveillance de la santé mentale positive

\section{FAMILLE}

2.1. Structure familiale
a. Parent seul
b. Contacts avec le parent biologique non cohabitant
c. Parents adolescents
d. Emprisonnement d'un parent

2.2. Relations familiales
a. Qualité et rapports des relations familiales
b. Repas en famille
c. Discussions en famille
d. Traitement par les parents/style parental

2.3. État de santé général de la famille
a. Bien-être mental de la famille
b. Problèmes fréquents de santé mentale des parents

2.4. Hygiène de vie parentale

a. Problèmes familiaux de dépendance

5. Composition du ménage

2.5. Aidant naturel

a. Aidant naturel pour un membre de la famille

6. Revenu du ménage

\section{COMMUNAUTÉ}

3.1. Capital social
a. Capital social

3.2. Soutien social, dispositions sociales et réseaux sociaux

1. Relations familiales

2. Style parental
a. Soutien social et dispositions sociales

b. Réseaux sociaux et contacts sociaux

3. État de santé physique et mentale de la famille

4. Consommation de substances des membres de la famille

3.3. Relations avec les pairs et les amis

a. Relations interpersonnelles

b. Engagement social

3.4. Inclusion et appartenance
a. Inclusion et exclusion sociales
b. Ménage au chômage
c. Éducation
d. Itinérance

3.5. Environnements scolaire et de travail
a. Environnement scolaire et réussite scolaire
b. Caractéristiques/environnement de travail
5. Environnement de travail

4. Environnement scolaire

3.6. Accès et organisation - soins de santé et services sociaux

a. Accès à des soins de santé, y compris en santé mentale

3.7. Participation
a. Participation

b. Bénévolat

3.8. Caractéristiques du quartier

a. Caractéristiques du quartier

b. Satisfaction à l'égard du quartier

3.9. Cohésion de la communauté

a. Cohésion de la communauté

b. Interdépendance de la communauté

3.10. Confiance et sécurité

a. Sécurité/criminalité/violence du quartier

b. Perception de la sécurité et de la criminalité

Suite page suivante 
TABLEAU 2 (suite)

Liste initiale et liste finale des indicateurs de surveillance de la santé mentale positive

\section{SOCIÉTÉ}

4.1. Justice sociale a. Justice sociale

4.2. Équité/égalité

a. Analyse de l'égalité

b. Pauvreté

4.3. Environnement physique (environnements bâti et naturel)

a. Lieux d'évasion

b. Espaces verts

c. État de la maison

d. Surpeuplement

e. Bruit

4.4. Politique et gouvernance

a. Politique publique favorisant la santé

4.5. Lois et politiques

a. Victimisation

b. Discrimination

4.6. Culture

a. Culture et valeurs

(Revenu du ménage, dans « Famille »)

1. Inégalité

(Environnement social du quartier et

Environnement bâti du quartier, dans « Communauté »)

2. Discrimination

3. Participation politique recevait un vote, et c'est la somme des votes attribués à chaque indicateur qui a servi au classement des indicateurs du plus populaire au moins populaire, classement qui a ensuite été vérifié par le groupe de travail.

Ce processus de sélection a permis de retrancher 5 indicateurs ayant obtenu peu de votes («tabagisme», «problèmes de jeu », « parents adolescents », « aidant naturel » et « engagement social et bénévolat») de la liste des 30 indicateurs, portant la liste à 25 indicateurs de déterminants. L'indicateur « engagement social et bénévolat » a finalement été réintroduit comme mesure de l'indicateur «implication communautaire» dans le niveau «communauté». Après nouvelle révision, l'indicateur « résilience et adaptation » a été divisé en 2 indicateurs pour plus de clarté, et 2 autres indicateurs ( « confiance » et « environnement social du quartier ») ont été regroupés en un seul, « environnement social du quartier », car ils se chevauchaient de façon significative au moment du choix des mesures.

Ainsi, 25 indicateurs ont été finalement choisis, relevant des 4 niveaux contextuels sélectionnés (« individu», «famille», « communauté », « société »).

Le tableau 2 permet de comparer la liste initiale et la liste finale d'indicateurs.

\section{Sélection des mesures}

Une fois les indicateurs choisis, nous avons procédé à une analyse des enquêtes basées sur la population canadienne afin de sélectionner des mesures pour chacun d'entre eux. Nous avons également pris en compte, au besoin, d'autres sources de données, en particulier certaines données géospatiales. Nous avons ensuite appliqué à ces mesures nos trois critères de sélection (exactitude, faisabilité et stabilité).

Avant de choisir les mesures, nous avons vérifié la pertinence des indicateurs en fonction des groupes d'âge. Comme certains indicateurs s'appliquent davantage à certaines périodes de la vie, nous avons séparé les mesures pour les enfants (0 à 11 ans), celles pour les jeunes (12 à 17 ans) et celles pour les adultes (18 ans et plus). Les indicateurs « expériences favorables dans l'enfance », « style parental » et « environnement scolaire » ont ainsi été appliqués aux enfants et aux jeunes seulement, alors que «environnement de travail » a été appliqué aux adultes uniquement. Le cadre portant sur les adultes est achevé (veuillez communiquer avec les auteurs pour en obtenir une copie). Les mesures pour le cadre portant sur les enfants et celui portant sur la jeunesse sont en cours d'élaboration.
Les résultats de notre analyse des enquêtes et d'autres sources de données nous ont permis de sélectionner des mesures et des sources de données susceptibles de rendre compte de tous les indicateurs choisis à l'échelle du Canada. Si aucune source de données ne s'est révélée valable, nous avons inclus des sources de données devenues inactives et pour lesquelles aucune nouvelle donnée ne sera donc publiée, de même que des sources portant sur certaines sous-populations uniquement. Nous n'avons pas seulement analysé les mesures relevant des enquêtes en cours sur la population canadienne, mais nous avons aussi consulté d'autres documents afin de disposer de mesures alternatives pour certains indicateurs, surtout pour ceux pour lesquels aucune source active (stable) de données n'était disponible.

Une consultation en ligne nous a permis de recueillir les conseils d'experts et d'intervenants sur les meilleures mesures à utiliser pour les indicateurs priorisés. Nous avons invité les groupes déjà consultés à participer, à savoir le Groupe de travail sur la promotion de la santé mentale et le Comité aviseur sur la surveillance de la santé mentale et des maladies mentales ainsi que les employés de l'Agence travaillant dans la surveillance et la promotion de la santé mentale. La première phase de la consultation a porté sur les mesures des 
résultats en matière de santé mentale positive et sur les mesures des déterminants du niveau contextuel « individu ». La seconde phase a porté sur les mesures des déterminants des trois autres niveaux contextuels : «famille», « communauté » et «société ». Chaque sondage présentait les mesures dégagées au moyen d'une analyse du contexte des enquêtes et des sources de données pour chacun des indicateurs de déterminants et de résultats en santé mentale positive, le cas échéant par période de parcours de vie (enfance, jeunesse, âge adulte). Les experts et les intervenants ont été invités à sélectionner les mesures en fonction de leur exactitude et de leur faisabilité. Disposer de données à jour (stables) était considérée comme une condition idéale, mais non nécessaire.

Les participants ont eu à répondre aux questions lors des sondages en fonction de trois modalités.

1) Si une seule mesure existante était envisagée comme source de données pour un indicateur, les participants avaient à donner leur avis sur l'utilisation de cette mesure pour le cadre et à suggérer d'autres échelles ou mesures validées pertinentes s'ils en connaissaient.

2) Si plusieurs mesures étaient disponibles pour un même indicateur, les experts étaient priés de choisir la mesure selon eux la plus adéquate pour cet indicateur, et de donner leur avis sur la pertinence et la disponibilité des mesures.

3) Si aucune mesure n'avait été relevée pour un indicateur, les experts étaient invités à en recommander une, et à fournir le cas échéant les sources de données correspondantes.

Nous avons sélectionné, en fonction des avis reçus, les mesures les plus pertinentes et les plus réalisables, en choisissant si possible celles provenant des mêmes sources de données. Si aucune source de données courantes n'avait été trouvée, nous avons sélectionné des mesures provenant d'enquêtes ponctuelles ou inactives, par exemple l'Enquête sur les jeunes Canadiens ou l'Enquête longitudinale nationale sur les enfants et les jeunes, utiles pour un premier bilan et dont le contenu peut être réutilisé pour des enquêtes à venir. Les mesures provenant de ces sources ont été considérées comme prioritaires lors de l'élaboration de données pour les bilans à venir.

Si plusieurs mesures étaient jugées à la fois exactes, réalisables et stables :

- les mesures bénéficiant une couverture nationale étaient privilégiées par rapport à celles assurant une couverture géographique partielle,

- les mesures issues d'enquêtes récentes étaient privilégiées par rapport à celles d'études plus anciennes,

- les mesures couvrant entièrement les périodes de parcours de vie des enfants, des jeunes et des adultes étaient privilégiées par rapport aux données portant sur une période plus limitée,

- les mesures testées et validées sur le plan psychométrique dans des enquêtes sur la population étaient privilégiées par rapport aux autres.

Si plusieurs mesures satisfaisaient à ces critères supplémentaires, celles provenant de l'Enquête sur la santé dans les collectivités canadiennes ont été privilégiées pour les adultes, afin de faciliter la modélisation et l'analyse des tendances. De même, les mesures provenant de l'Étude sur les comportements de santé des jeunes d'âge scolaire ou de l'Enquête sur la santé dans les collectivités canadiennes ont été privilégiées pour les jeunes par rapport à d'autres sources de données.

Lors de chaque phase de consultation, l'inclusion des mesures ou des sources de données supplémentaires suggérées par les intervenants a fait l'objet de vérifications, afin que le cadre intègre les éléments les plus probants sur la santé mentale positive et ses facteurs déterminants. Certaines lacunes en matière de données ont été relevées, lorsque aucune mesure n'était disponible ou lorsque les mesures étaient de piètre qualité, et l'Agence collabore de façon proactive avec ses partenaires afin de mettre en branle les mécanismes permettant de combler les plus criantes de ces lacunes.

L'Agence a préparé deux rapports de synthèse pour présenter les principaux résultats des consultations aux intervenants, le premier traitant du cadre portant sur les adultes et le second des cadres portant sur les enfants et sur les jeunes.

\section{Conclusions et répercussions}

Le Cadre d'indicateurs de surveillance de la santé mentale positive constitue le socle sur lequel repose la sélection des indicateurs et des mesures permettant des bilans sur la santé mentale positive des Canadiens. Ces indicateurs dressent un portrait d'ensemble des résultats au Canada en matière de santé mentale positive et de ses facteurs déterminants clés chez les enfants, chez les jeunes et chez les adultes. En tout, 5 indicateurs de résultats en santé mentale positive et 25 indicateurs connexes, relevant des niveaux contextuels « individu», «famille», « communauté » et « société », ont été sélectionnés et, pour les adultes, les mesures correspondantes ont déjà été intégrées. Les analyses psychométriques indiquent que la démarche proposée pour rendre compte des résultats en santé mentale positive a une validité empirique $^{26}$. Les mesures pour les cadres portant sur l'enfance et sur la jeunesse sont en cours de sélection, et les données pour le cadre portant sur les jeunes devraient être publiées en 2016.

Ce travail permet le renforcement de la promotion de la santé mentale positive en tant qu'activité de santé publique d'importance, et il comble les lacunes statistiques majeures relevées dans la Stratégie canadienne en matière de santé mentale pour le Canada. En fournissant un aperçu de la santé mentale positive chez les Canadiens, ce cadre peut servir de référence à bien des niveaux lors de l'établissement des politiques et des programmes de promotion de la santé mentale et de prévention des maladies mentales. Les écarts de niveaux constatés en matière de santé mentale positive pourraient aider à l'identification des groupes susceptibles de tirer profit d'interventions, tandis que la modélisation des facteurs de risque et de protection fournirait plutôt un guide sur la nature des interventions à mener. L'accumulation des données au cours du temps nous permettra d'observer au fur et à mesure les changements qui s'opèrent dans le portrait de la santé mentale positive des Canadiens.

L'un des succès de ce travail a été de préconiser une démarche coopérative, 
favorisant l'établissement de relations étroites entre les intervenants. La création du Cadre a nécessité la collaboration et la consultation d'instances gouvernementales provinciales et territoriales, d'organismes indépendants (dont la CSMC) et de chercheurs. Le cadre tient ainsi compte à la fois des besoins des intervenants et des meilleures données probantes en santé mentale positive, et il est en mesure de fournir un support d'aide à la décision en matière de recherche, de programmes et de politiques.

La capacité à faire des bilans reposant sur ces indicateurs et ces mesures constitue une priorité. On peut déjà consulter le premier résumé infographique sur la santé mentale positive en ligne à la page http://www. phac-aspc.gc.ca/mh-sm/mhp-psm/pmh-smpfra.php. L'édition 2016 sur les adultes des Statistiques rapides du Cadre d'indicateurs de surveillance de la santé mentale positive est également publiée dans ce numéro. Ces statistiques fournissent les plus récentes données sur les résultats et les facteurs de risque et de protection en matière de santé mentale positive. En 2016, une infobase sera mise en ligne pour que les utilisateurs explorent les données du cadre en fonction de quelques variables sociodémographiques clés, notamment l'âge, le sexe, le revenu et le statut d'immigration. Une attention particulière sera portée à l'élaboration de données comblant les principales lacunes statistiques constatées et assurant une amélioration constante des indicateurs et des mesures sélectionnés. Des travaux sont en cours pour élaborer un cadre de surveillance similaire pour le suicide et ses facteurs de risque et de protection, dont bon nombre sont les mêmes que ceux influençant la santé mentale positive. D'autres travaux pourraient porter sur les mesures à ajouter dans le cadre de la Stratégie en matière de santé mentale pour le Canada, notamment sur les maladies mentales et le suicide.

\section{Remerciements}

Nous tenons à remercier le Comité aviseur sur la surveillance de la santé mentale et des maladies mentales pour ses avis et ses conseils d'expert sur la sélection des indicateurs et des mesures, Marianna Ofner pour la direction initiale des travaux sur l'élaboration du cadre conceptuel de surveillance de la santé mentale positive et l'Unité de la promotion de la santé mentale pour sa collaboration soutenue à notre travail de surveillance.

\section{Références}

1. Agence de la santé publique du Canada. Promotion de la santé mentale: Promouvoir la santé mentale, c'est promouvoir le meilleur de nous même [Internet]. Ottawa (Ont.) : Agence de la santé publique du Canada [modification le 6 mai 2014; consultation le 26 septembre 2015]. Consultable en ligne à la page : http://www.phac-aspc.gc.ca/mh-sm/ mhp-psm/index-fra.php

2. Organisation mondiale de la santé. Dix faits sur la santé mentale [Internet]. Genève (Suisse) : Organisation mondiale de la santé [mise à jour en août 2014; consultation le 26 septembre 2015]. Consultable en ligne à la page : http://www.who.int/features/fact files/mental_health/fr/

3. Agence de la santé publique du Canada. Évaluation de la fonction de surveillance au sein de l'Agence de la santé publique du Canada [Internet]. Ottawa (Ont.) : Agence de la santé publique du Canada [modification le 14 mai 2013; consultation le 26 septembre 2015]. Consultable en ligne à la page : http://www.phac-aspc.gc.ca/about_apropos/ evaluation/reports-rapports/2012-2013/sf-fs/ section-1-fra.php

4. Organisation mondiale de la santé. Public Health Surveillance. Genève (Suisse) : Organisation mondiale de la santé [mise à jour en 2015; consultation le 26 septembre 2015]. Consultable en ligne à la page : http://www.who. int/topics/public_health_surveillance/en/

5. Commission de la santé mentale du Canada. Changer les orientations, changer des vies. Calgary (Alberta) : Commission de la santé mentale du Canada; 2012. Pdf (11,5 Mo) téléchargeable à partir du lien : http://www. mentalhealthcommission.ca/Francais/system/files/private/document/MHStrategy_ Strategy_FRE.pdf

6. Agence de la santé publique du Canada; Société pour les troubles de l'humeur du Canada; Statistique Canada; Institut canadien d'information sur la santé. Aspect humain de la santé mentale et de la maladie mentale au Canada 2006. Ottawa (Ont.) : Gouvernement du Canada; 2006. Consultable en ligne à la page : http://www.phac-aspc.gc.ca/publicat/ human-humain06/index-fra.php
7. Waddell C, Shepherd CA, Chen A, Boyle MH. Creating comprehensive children's mental health indicators for British Columbia. Can J Commun Ment Health. 2013;32(1):9-27.

8. Parkinson J. Establishing national mental health and well-being indicators for Scotland. J Public Mental Health. 2007;5(1):42-48.

9. Korkeila J, Lehtinen V, Bijl R, Dalgard OS, Kovess V, Morgan A et collab. Establishing a set of mental health indicators for Europe. Scand J Public Health. 2003;31 (6):451-459.

10. McLeroy KR, Bibeau D, Steckler A, Glanz K. An ecological perspective on health promotion programs. Health Educ Q. 1988; 14(4):351-377.

11. Dahlgren G, Whitehead M. Policies and strategies to promote equity in health. Stockholm (Suède) : Institute for Futures Studies; 1991.

12. Centers for Disease Control and Prevention. The social-ecological model: a framework for prevention [Internet]. Bethseda (Maryland) : Centers for Disease Control and Prevention [mise à jour le 25 mars 2015; consultation le 26 septembre 2015]. Consultable en ligne à la page : http://www.cdc. gov/violenceprevention/overview/socialecologicalmodel.html

13. Organisation mondiale de la santé, Department of Mental Health and Substance Abuse; Victorian Health Promotion Foundation; University of Melbourne. Promoting mental health: concepts, emerging evidence, practice: summary report. Genève (Suisse) : Organisation mondiale de la santé; 2004

14. Keating DP, Hertzman C (dir.). Developmental health and the wealth of nations: social, biological and educational dynamics. New York (New York) : The Guilford Press; 1999.

15. Hall HI, Correa A, Yoon PW, Braden CR. Lexicon, definitions, and conceptual framework for public health surveillance. MMWR Surveill Summ. 2012;61 (Suppl):10-14.

16. Betancourt MT, Roberts KC, Bennett TL, Driscoll ER, Jayaraman G, Pelletier L. Surveillance des maladies chroniques au Canada : Cadre conceptuel d'indicateurs des maladies chroniques. Maladies chroniques et blessures au Canada. 2014;34(1):1-30. 
17. Flowers J, Hall P, Pencheon D. Public health indicators. Public Health. 2005;119(4):239-245.

18. Hermann CR, Leff HS, Lagodmos G. Selecting process measures for quality improvement in mental healthcare. Cambridge (Massachuset) : Health Services Research Institute; 2002. Publication conjointe avec le Center for Mental Health Services.

19. Australian Institute of Health and Welfare. Key indicators of progress for chronic disease and associated determinants. Canberra (Australie) : Australian Institute of Health and Welfare; 2009.

20. National Mental Health Performance Subcommittee. Key performance indicators for Australian public mental health services. $2^{\mathrm{e}}$ éd. Canberra (Australie) : Commonwealth d'Australie; 2011.

21. Deci EL, Ryan RM. Hedonia, eudaimonia, and well-being: an introduction. J Happiness Stud. 2008;9(1):1-11.

22. Huppert FA, So TT. Flourishing across Europe: application of a new conceptual framework for defining well-being. Soc Indic Res. 2013;110(3):837-861.

23. Lakaski C, Trottier M. Élaboration d'une définition opérationnelle de la santé mentale positive. Rapport final fondé sur une consultation et un atelier tenus les 26 et 27 février 2009, Conference Centre, Ottawa, Ontario. Lieu : Ottawa (Ontario) : Unité de la promotion de la santé mentale; 2009. (rapport non publié)

24. LeMessurier J, O’Donnell S, Walsh P, McRae L, Bancej C. L'élaboration d'indicateurs nationaux pour la surveillance de l'ostéoporose au Canada. Maladies chroniques et blessures au Canada. 2012;32(2):101-107.

25. Réseau pancanadien de santé publique. Au sujet du Réseau pancanadien de santé publique [Internet]. Ottawa (Ont.) : Réseau pancanadien de santé publique [modification le 9 juillet 2012; consultation le 26 septembre 2015]. Consultable en ligne à la page : http://www.phn-rsp.ca/network-fra.php

26. Orpana H, Vachon J, Dykxhoorn J, Jayaraman G. Measuring positive mental health using items from the Mental Health Continuum-Short Form in the Canadian population: a new approach. Rapport interne. 2015 ou 2016; [sous presse]. 